\title{
Isolated Bone Marrow Mastocytosis may be Associated with Reduced Overall Survival
}

\author{
Severino-Freire $M^{1}$, Damaj GL ${ }^{2}$, J endoubi $F^{1}$, \\ Negretto $M^{1}$, Laurent $C^{3}$, De Mas $V^{4}$, Evrard $S^{3}$, \\ Apoil PA ${ }^{5}$, Mailhol $\mathbf{C}^{6}$, Degboe $Y^{7}$, Dubreuil $\mathbf{P}^{8}$, \\ Hermine $\mathrm{O}^{9}$, Paul $\mathrm{C}^{1}$ and Livideanu $\mathrm{CB}^{1 *}$ \\ ${ }^{1}$ Mastocytosis Expert Center (CEREMAST) Toulouse, \\ Department of Dermatology, Toulouse University \\ Hospital, Paul Sabatier University, France \\ ${ }^{2}$ Departement of Haematology, Caen University Hospital, \\ France \\ ${ }^{3}$ Department of Anatomy and Pathological Cytology, \\ Toulouse University Hospital, Paul Sabatier University, \\ CRCT INSERM U1037, CEREMAST Toulouse, Toulouse, \\ France \\ ${ }^{4}$ Department of Haematology, CEREMAST Toulouse, \\ Toulouse, France \\ IImmunology Clinical Laboratory, CEREMAST Toulouse, \\ Toulouse, France \\ ${ }^{6}$ Department of Pneumo-Allergology, CEREMAST \\ Toulouse, Toulouse, France \\ 'Department of Rheumatology, CEREMAST Toulouse, \\ Toulouse, France \\ ${ }^{8}$ CRCM, (CEREMAST, Marseille, Equipe Labellisée Ligue \\ Contre le Cancer), Inserm, Institut Paoli-Calmettes, Aix- \\ Marseille University, CNRS, Marseille, France \\ ${ }^{9}$ Haematology, CEREMAST Necker, AP-HP, Paris, France \\ *Corresponding author: Cristina Bulai Livideanu, \\ CEREMAST Toulouse, Department of Dermatology, \\ Toulouse University Hospital, Paul Sabatier University, \\ France
}

Received: October 01, 2021; Accepted: October 29, 2021; Published: November 05, 2021

\section{Short Communication}

Mastocytosis is characterized by accumulation/proliferation of abnormal Mast Cells (MCs) in tissues [1]. Depending on organ involvement, two main forms of mastocytosis are identified Isolated Cutaneous Mastocytosis (ICM), when the skin is the only tissue affected $[2,3]$ and Systemic Mastocytosis (SM), characterized by MC infiltrates in internal organs, mostly the Bone Marrow (BM). SM can be or not associated with concomitant skin involvement. The most frequent form of SM is indolent SM [4].

Isolated Bone Marrow Mastocytosis (IBMM) constitutes a variant of indolent SM described in the 2008 WHO classification for mastocytosis [5]. There are limited information on the course and prognosis of IBMM in the literature. In the literature, it exist for patients with non-advanced and advanced mastocytosis a score who can be used to predict survival outcomes even if the predictive value of the International Prognostic Scoring system for Mastocytosis (IPSM) needs to be confirmed in forthcoming trials [6].

IBMM represents a diagnostic challenge for clinicians as clinical manifestations such as anaphylaxis; osteoporosis and digestive symptoms are not specific.

The main objective of this study was to analyze the overall survival of patients with IBMM in adults with SM. The secondary objectives were to assess the frequency of IBMM in adults with SM, compare the clinical and laboratory characteristics of patients IBBM to those of patients with SM associated cutaneous mastocytosis (SMcm) and evaluate the IPSM prognostic score in our cohort.

Consecutive adult patients with a diagnosis of SM evaluated between January 2009 and January 2020 were included. All patients were prospectively included in the study supported by «French Association for the Initiatives of Research on Mastocyte and Mastocytosis» and entitled "Physiopathological and clinical study of mastocytosis in adult patients". The study was approved by the Institutional Review Board of Necker Enfants-Malades Hospital on $8^{\text {th }}$ November 2000. The study was carried out in accordance with the Declaration of Helsinki. Written informed consent was obtained from all patients. The diagnosis of SM was made according to the diagnostic criteria defined by the World Health Organization (WHO) $[4,5,7,8]$. The diagnosis of IBMM was made by one of the board dermatologists with expertise in mastocytosis (MSF, FJ, CP, and/or CBL) after full body examination ruling out visible signs of mastocytosis (Figure 1 and Table 1).

We included 193 adult patients with SM. We diagnosed 38 patients with IBMM (19.6\%). The duration of follow-up was 300 months (IQR: 24-128). Regarding the overall survival, there were six deaths in the IBMM group (15.7\%) and none in SMcm population $(\mathrm{p}<0.01)$ (Figure 1). Among these patients, four deaths were related to the mastocytosis associated haematological disorder and the latter two were due to lung cancer and septic shock. The sex ratio was similar in both groups with $44.5 \%$ of male in SMcm population and $44.7 \%$ in IBMM group. IBMM patients were significantly older than SMcm patients (56 years (IQR: 42.5-61.8) versus 46 years (IQR: $35-$ 56.8) $(\mathrm{p}=0.02)$. The average of the delay of diagnosis between first manifestations and diagnosis was significant different between two groups: 3 months (IQR: 1-5.5) in IBMM population versus 5 months

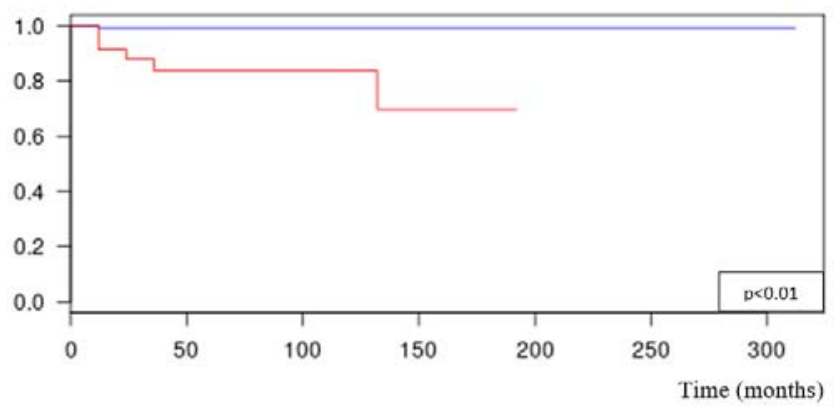

Figure 1: Overall survival IBMM versus $\mathrm{SMcm}$. 
Table 1: Mast cell mediator-related symptoms in two populations.

\begin{tabular}{|l|c|c|c|}
\hline & SMcm & IBMM & p-value \\
\hline Cutaneous symptoms & $115(74.2)$ & $17(44.7)$ & 0.0004 \\
\hline Flush & $72(46.5)$ & $11(29)$ & 0.05 \\
\hline Pruritus & $64(41.3)$ & $9(23.7)$ & 0.04 \\
\hline Darier's sign & $75(48.4)$ & $1(2.6)$ & $<0.01$ \\
\hline Superficial urticaria & $2(1.3)$ & $4(10.5)$ & $<0.01$ \\
\hline Angioedema & $5(3.2)$ & $8(21.0)$ & $<0.01$ \\
\hline Gastro-intestinal symptoms & $84(54.2)$ & $20(52.6)$ & 0.83 \\
\hline Diarrhea & $70(45.2)$ & $16(42.1)$ & 0.73 \\
\hline Abdominal cramping & $70(45.2)$ & $11(29.0)$ & 0.06 \\
\hline Nausea & $15(9.7)$ & $7(18.4)$ & 0.12 \\
\hline Cardiovascular symptoms & $49(31.6)$ & $18(47.4)$ & 0.06 \\
\hline Syncope & $21(13.6)$ & $7(18.4)$ & 0.44 \\
\hline Hypotension & $24(15.5)$ & $7(18.4)$ & 0.65 \\
\hline All shock & $26(16.7)$ & $14(36.8)$ & 0.006 \\
\hline Mixed shock & $4(2.5)$ & $1(2.6)$ & 0.98 \\
\hline Idiopathic shock & $18(11.8)$ & $9(23.7)$ & 0.05 \\
\hline Hymenoptera shock & $10(6.5)$ & $5(13.2)$ & 0.16 \\
\hline Allergic shock & $12(7.7)$ & $6(15.8)$ & 0.12 \\
\hline Urinary symptoms & $23(14.8)$ & $2(5.3)$ & 0.12 \\
\hline Respiratory symptoms & $14(9)$ & $7(18.4)$ & 0.09 \\
\hline Cough & $2(1.3)$ & $0(0)$ & 0.48 \\
\hline Dyspnea & $7(18.7)$ & $2(5.3)$ & 0.04 \\
\hline Depression & $3(7.9)$ & 0.07 \\
\hline Headache & $16.3)$ & (15) \\
\hline
\end{tabular}

(IQR: 1-10.3) in SMcm group ( $\mathrm{p}=0.001)$.

Regarding mast cells mediator-related symptoms (mcms), superficial spontaneous urticaria $(10.5 \%$ vs. $1.3 \%, \mathrm{p}=0.03)$ and angioedema $(21 \%$ vs. $3.2 \%, \mathrm{p}<0.01)$ were more frequent in IBMM as compared to SMcm patients. Similarly, anaphylactic shock, was more frequent in IBBM than SMcm patients $(36.8 \%$ vs. $16.7 \%, \mathrm{p}=$ $0.006)$, especially idiopathic anaphylactic shock subtypes $(23.7 \%$ vs. $11.6 \%, \mathrm{p}=0.05$ ). In IBMM group the dyspnea was also more frequent $(18.4 \%$ vs. $9 \%, \mathrm{p}=0.04)$ than in $\mathrm{SMcm}$ group. By contrast, $\mathrm{SMcm}$ patients exhibited a higher frequency of other symptoms related to skin mast cells than IBMM patients flush $(46.5 \%$ vs. $28.9 \%, \mathrm{p}=0.05)$, and pruritus $(41.3 \%$ vs. $23.7 \%, \mathrm{p}=0.04)$.

Regarding others mcms, there was no significant difference between the two groups (Table 1). There was no significant difference between the two populations concerning WHO criteria of SM, biological parameters, frequency of other neoplastic hematologic disease (myelodysplastic syndrome, polycythemia, leukemia, lymphoma...) and bone marrow features, including bone fracture (data not shown).

The IPSM was statistically significant more important in the IBBM group vs SMcm group (mean: $0.8 v s .-0.6, \mathrm{p}<0.001$ ).

To our knowledge, this is the first study showing a significant difference in terms of overall survival in IBMM vs $S M c m$ patients. Indeed more of observed deaths in IBMM patients were related to a haematological neoplasm associated with mastocytosis. The IPSM score validated the data found in our cohort.

Moreover, we show that $19.6 \%$ of our adult cohort with SM presented IBBM. These patients showed higher prevalence of cutaneous symptoms such as superficial urticarial, angioedema and, anaphylactic shock especially idiopathic ones. These results underscores the importance of screening this population for the diagnostic criteria defined by the WHO in order to optimize their care, especially in case of idiopathic anaphylactic shock.

The frequency of IBBM patients varies between 10.5 to $54.7 \%$ according to the literature data [9-13]. Alvarez-Twose I et al., [11] showed a frequency of $29.6 \%$ cases of IBMM (48 cases of out 162 all SM) in the Spanish population. Lim et al., found a frequency of $10.5 \%$ patients with IBMM ( 36 of out 342 all SM) in American population [9]. Zanotti $R$ et al. [12] showed a frequency of IBMM of $54.7 \%$ (46 of out 84 patients) among indolent SM Italian patients. This frequency is probably overestimated because authors selected patients who presented unexplained/recurrent anaphylaxis or severe allergic reactions to hymenoptera stings with persistent elevated serum tryptase level. More recently, European Competence Network on Mastocytosis [13] showed a prevalence of $23.7 \%$ of IBMM among all mastocytosis patients without advanced SM.

What emerges from the literature and in our study is the prevalence of anaphylaxis and/or severe anaphylaxis like as anaphylactic shock in IBMM patients. In fact, in the series of Lim et al., $78 \%$ of them had history of anaphylactic reactions. In the series of Zanotti et al., 95.6\% of IBMM had anaphylaxis, whose $91.3 \%$ after hymenopter sting. In our cohort, the frequency of anaphylactic shocks was also higher in IBBM than SMcm patients. That might explain the delay of diagnosis between first manifestations and diagnosis least important in IBBM than SMcm patients. Therefore, IBMM patients seem distinct from other SM patients in terms of anaphylactic shock's risk [14,15]. A quickly diagnostic could also allow us to ensure them optimal care. For example, patients with anaphylactic shock to hymenoptera venom should be considered for life-long venom immunotherapy $[15,16]$, and the patients with idiopathic anaphylactic shock may also be effectively treated with omalizumab [17].

Our study indicates that IBMM is associated with a higher frequency of life-threatening symptoms and reduced survival. Collaborative longitudinal studies are needed to better evaluate these patients and improve quality of care.

\section{References}

1. Valent $P$, Akin C, Sperr WR, et al. Diagnosis and treatment of systemic mastocytosis: state of the art. Br J Haematol. 2003; 122: 695-717.

2. Hartmann K, Escribano L, Grattan C, et al. Cutaneous manifestations in patients with mastocytosis: consensus report of the European Competence Network on Mastocytosis; the American Academy of Allergy, Asthma \& Immunology; and the European Academy of Allergology and Clinical Immunology. J Allergy Clin Immunol. 2016; 137: 35-45.

3. Matito A, Azaña JM, Torrelo A, et al. Cutaneous Mastocytosis in Adults and Children: New Classification and Prognostic Factors. Immunol Allergy Clin North Am. 2018; 38: 351-363.

4. Valent $\mathrm{P}$, Akin C, Escribano L, et al. Standards and standardization 
in mastocytosis consensus statements on diagnostics, treatment recommendations and response criteria. Eur J Clin Invest. 2007; 37: 435-453.

5. Horny HP, Akin C, Metcalfe DD, et al. World Health Organization (WHO) classification of tumours pathology and genetics of tumours of the haematopoietic and lymphoid tissues. In: Swerdlow SH, Campo PE, Harris NL, Jaffe ES, Pileri SA, Stein H, editors. Lyon, France. 2008: 54-63.

6. Sperr WR, Kundi M, Alvarez-Twose I, et al. International prognostic scoring system for mastocytosis (IPSM): a retrospective cohort study. Lancet Haematol. 2019; 6: e638-e649.

7. Valent P, Sperr WR, Schwartz LB, et al. Diagnosis and classification of mast cell proliferative disorders: delineation from immunologic diseases and nonmast cell hematopoietic neoplasms. J Allergy Clin Immunol. 2004; 114: 3-11.

8. Valent $\mathrm{P}$, Horny HP, Li CY, et al. World Health Organization (WHO) classification of tumours pathology and genetics of tumours of the haematopoietic and lymphoid tissues. In Lyon FRANCE: Jaffe ES, Harris NL, Stein H, Vardiman JW, editors. 2001: 291-302.

9. Lim KH, Tefferi A, Lasho TL, et al. Systemic mastocytosis in 342 consecutive adults: survival studies and prognostic factors. Blood. 2009; 113: 5727-5736.

10. Pardanani A, Lim $\mathrm{KH}$, Lasho $\mathrm{TL}$, et al. WHO subvariants of indolent mastocytosis: clinical details and prognostic evaluation in 159 consecutive adults. Blood. 2010; 115: 150-151.
11. Alvarez-Twose I, González de Olano D, Sánchez-Muñoz L, et al. Clinical, biological, and molecular characteristics of clonal mast cell disorders presenting with systemic mast cell activation symptoms. J Allergy Clin Immunol. 2010; 125: 1269-1278.

12. Zanotti $R$, Bonadonna $P$, Bonifacio $M$, et al. Isolated bone marrow mastocytosis: an underestimated subvariant of indolent systemic mastocytosis. Haematologica. 2011; 96: 482-484.

13. Trizuljak J, Sperr WR, Nekvindová L, et al. Clinical features and survival of patients with indolent systemic mastocytosis defined by the updated WHO classification. Allergy. 2020; 75: 1927-1938.

14. Alvaro-Lozano M, Akdis CA, Akdis M, et al. EAACI Allergen Immunotherapy User's Guide. Pediatr Allergy Immunol. 2020; 31: 1-101.

15. Schuch A, Brockow K. Mastocytosis and Anaphylaxis. Immunol Allergy Clin North Am. 2017; 37: 153-164.

16. Gruzelle V, Ramassamy M, Bulai Livideanu C, et al. Safety of ultra-rush protocols for hymenoptera venom immunotherapy in systemic mastocytosis. Allergy. 2018; 73: 2260-2263.

17. Jendoubi F, Gaudenzio N, Gallini A, et al. Omalizumab in the treatment of adult patients with mastocytosis: A systematic review. Clin Exp Allergy. 2020; 50: 654-661. 